\title{
Germanica
}

\section{Historiographie und Metahistoriographie im Roman Magma von Michael Stavarič}

Historiography and metahistoriography in Michael Stavaričs Magma

Historiographie et métahistoriographie dans le roman Magma de Michael

Stavarič

Johann Georg Lughofer

\section{CpenEdition}

Journals

Édition électronique

URL : http://journals.openedition.org/germanica/1995

DOI : 10.4000/germanica. 1995

ISSN : 2107-0784

Éditeur

Université de Lille

Édition imprimée

Date de publication : 31 décembre 2012

Pagination : 89-101

ISBN : 9782913857308

ISSN : 0984-2632

Référence électronique

Johann Georg Lughofer, « Historiographie und Metahistoriographie im Roman Magma von Michael Stavarič », Germanica [Online], 51 | 2012, Online erschienen am: 14 Januar 2013, abgerufen am 06 Oktober 2020. URL : http://journals.openedition.org/germanica/1995 ; DOI : https://doi.org/10.4000/ germanica.1995

Ce document a été généré automatiquement le 6 octobre 2020.

(C) Tous droits réservés 


\title{
Historiographie und
} Metahistoriographie im Roman Magma von Michael Stavarič

\author{
Historiography and metahistoriography in Michael Stavarič's Magma \\ Historiographie et métahistoriographie dans le roman Magma de Michael \\ Stavarič
}

Johann Georg Lughofer

Dieser Beitrag analysiert die Geschichtsbetrachtung in Michael Stavarič' Roman Magma (2008), der in seinem Kern eine Narrative von historischen Ereignissen darstellt. Deren Auswahl und Aneinanderreihung sowie die verwendeten Stilmittel lassen eine eigene Historiographie und deren Reflexion entstehen. Nach einer kurzen Präsentation des Autors, der aufgrund seiner Biographie in den Kontext der interkulturellen Literatur einzuordnen ist, soll diesen Aspekten textorientiert nachgegangen werden.

\section{Der „intellektuelle shooting star“ Michael Stavarič}

Siebenjährig kam Stavarič nach Österreich, da seine Eltern aus der damaligen kommunistischen Tschechoslowakei ausgewandert waren. Sein Studium der Bohemistik und Publizistik schloss er 1998 mit einer Diplomarbeit zu Sprachstrukturen tschechischer Schlagzeilen ab, wonach er als Rezensent bei Der Presse und der Wiener Stadtzeitung Falter sowie bei verschiedenen Verlagen als Experte für tschechische Literatur tätig war. Der umtriebige Intellektuelle übersetzte nicht nur aus dem Tschechischen, lehrte Inline-Skating an der Sportuniversität Wien, sondern war auch Executive Coordinator des Präsidenten des Internationalen P.E.N.-Klubs und Sekretär des mittlerweile verstorbenen tschechischen Botschafters a. D. und Schriftstellers Jiri Gruša. Heute lebt er als freier Schriftsteller in Wien.

In biographischen und journalistischen Kommentaren zu Stavarič sind immer wieder Begriffe wie „intellektueller Shootingstar“ $z u$ finden ${ }^{1}$, eine Zuschreibung, die leicht 
verständlich wird, wenn man sich die Vielzahl der ihm zugesprochenen bedeutenden Auszeichnungen und Preise vor Augen führt: Allein im Frühjahr 2012 erhielt Stavarič u.a. den Adelbert-von-Chamisso-Preis sowie den Österreichischen Staatspreis für Jugend- und Kinderliteratur ${ }^{2}$.

\title{
Klees Angelus Novus bei Walter Benjamin, Laurie Anderson und Michael Stavarič
}

Schon das einführende Motto macht den Schwerpunkt des Romans deutlich, nämlich eine alternative Geschichtsschreibung. Das Motto ist ein Zitat aus Laurie Andersons Song The Dream before: „History is an angel being blown backwards into the future / History is a pile of debris / The angel wants to go back and fix things to repair / The things that have been broken / But there is a storm blowing from Paradise / The storm keeps blowing the angel

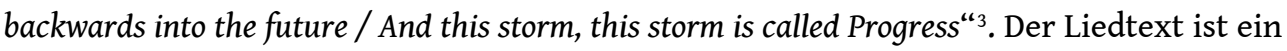
Zitat von Walter Benjamins neunter These Über den Begriff der Geschichte, das offensichtlich durch Vereinfachung, Übersetzung und Liedform gebrochen ist. In dieser neunten These erläutert Benjamin das Bild Angelus Novus von Paul Klee symbolisch und erkennt darin einen „Engel der Geschichte“, der mit Entsetzen auf etwas starrt, von dem er sich entfernt:

\begin{abstract}
„Der Engel der Geschichte muß so aussehen. Er hat das Antlitz der Vergangenheit zugewendet. Wo eine Kette von Begebenheiten vor uns erscheint, da sieht er eine einzige Katastrophe, die unablässig Trümmer auf Trümmer häuft und sie ihm vor die Füße schleudert. Er möchte wohl verweilen, die Toten wecken und das Zerschlagene zusammenfügen. Aber ein Sturm weht vom Paradiese her, der sich in seinen Flügeln verfangen hat und so stark ist, daß der Engel sie nicht mehr schließen kann. Dieser Sturm treibt ihn unaufhaltsam in die Zukunft, der er den Rücken kehrt, während der Trümmerhaufen vor ihm zum Himmel wächst. Das, was wir den Fortschritt nennen, ist dieser Sturm. "
\end{abstract}

Dieses starke, lyrisch anmutende Bild wurde zum bekanntesten Teil der sogenannten Geschichtsphilosophischen Thesen, welche als letzte Aufzeichnungen des sich auf der Flucht befindlichen Benjamins zwar ausdrücklich nicht für den Druck bestimmt waren, aber nach der posthumen Veröffentlichung 1941 weltbekannt wurden. Epochale Bedeutung wurde ihnen in den Zeiten der großen marxistischen Theoriebildungen der sechziger und siebziger Jahre des letzten Jahrhunderts zugesprochen ${ }^{5}$. Es ist eine unvollendete, fragmentarische, heterogene, zum Teil widersprüchliche Sammlung von rätselhaften Orakelreden und Parabeln über Theologie bis zur bodenständig formulierten klassenkämpferischen Stellungnahme, von Hymnen an den Historischen Materialismus bis zur theoretisch fundierten Kritik am marxistischen Fortschrittsbegriff.

Das Motto des Romans soll nebst den weiteren Thesen Benjamins als Ausgangspunkt und Folie dienen, um die zentralen Aspekte der Metahistoriographie im Roman aufzuzeigen. Die Parallelen zu Benjamin ermöglichen eine aufschlussreiche Einordnung der Geschichtsbetrachtung im Roman. 


\section{Überlieferung der Kultur als Überlieferung der Barbarei}

7 Benjamin brandmarkt in seiner siebten These historistische Verfahren, die durch Einfühlung gekennzeichnet sind. Dabei fühlen sich die Geschichtsschreiber „unweigerlich in die Sieger" ein. „Die jeweils Herrschenden sind aber die Erben aller, die je gesiegt haben. Die Einfühlung kommt demnach den jeweils Herrschenden allemal zugut." Sogenannte Kulturgüter seien nichts anderes als Beute im brutalen Triumphzug der Herrschenden. Nach Benjamin werden sie von historischen Materialisten mit Distanz betrachtet. Neben den großen Genies habe immer auch die „namenslose Fron ihrer Zeitgenossen“ unser Dasein geschaffen. Die traditionelle Geschichtsschreibung vergesse dieses Leid. So folgert Benjamin:

„Es ist niemals ein Dokument der Kultur, ohne zugleich ein solches der Barbarei zu sein. Und wie es selbst nicht frei ist von Barbarei, so ist es auch der Prozess der Überlieferung nicht, in der es von dem einen an den andern gefallen ist. Der historische Materialist rückt daher nach Maßgabe des Möglichen von ihr ab. Er betrachtet es als seine Aufgabe, die Geschichte gegen den Strich zu bürsten." ${ }^{6}$

Obwohl sich der Roman keineswegs linientreu im Rahmen eines historischen Materialismus bewegt, folgt er Benjamins Forderung dahingehend, dass er die Geschichte gehörig „gegen den Strich bürstet“. Der Roman ist als monologisierende Erzählung aufgebaut, in der ein Zoohändler von verschiedensten Ereignissen unterschiedlichster Epochen berichtet, die er zumeist als Augenzeuge, oft gar als Insider miterlebt $\mathrm{zu}$ haben scheint. Gewechselt wird in hohem Tempo sprunghaft zwischen den rhythmischen Blöcken, Erinnerungen und Assoziationen. Die Sprache ist dabei einmal bibelhaft, ein andermal umgangssprachlich, ja kumpelhaft. Sie belehrt, zitiert aus "hoher" Literatur, verbreitet Anekdoten oder bringt Seemannslieder vor. Soviel ist zitiert und montiert, soviel wird gesprochen, dass die Kursivsetzung zumeist die Funktion der Hervorhebung solcher Stellen anstatt der Markierung der direkten Rede übernimmt.

Dass die Lesenden dieser Überlieferung nicht trauen dürfen und in dieser Überlieferung Barbarei steckten könnte, wird dabei nahegelegt, denn der Text spielt mehrfach darauf an, dass der Erzähler der Teufel selbst sei. Dieser wirkt dabei aber weder sehr mächtig, noch sehr böse. Textstellen erinnern spielerisch an Goethes Mephisto, an seine bekannten Zeilen wie „Ich bin die Kraft, die stets das Böse will und stets das Gute schafft.“ - beispielsweise: „Dass ich mich redlich mühte, Gutes zu tun? Dass ich den Menschen eine Muse war, die innere Stimme der unbestechlichen Vernunft, ein guter Geist mit bösen Fängen?““ (M 179)

Der Lesende bleibt im Ungewissen, wer bzw. wie dieser Geschichte schreibende Teufel denn sei. Mehrdeutige und verwirrende Stellen werden eingeschoben: manchmal erscheint der Zoohändler gar messianisch, so zum Beispiel wenn er Tiere verkauft und das schöne Gefühl hat, ,jemand schaut über meine Schultern und sagt: Gut gemacht, Sohn! ${ }^{\omega_{7}}$ (M 108) Er spricht von gewünschter Weltverbesserung und bringt es nicht übers Herz, die geerbten Pflanzen seines verstorbenen Freundes zu entsorgen. Als Zoohändler liebt er Tiere und quält sich mit Bedenken moralischer Natur, beispielsweise wenn er diese in Käfige steckt und verkauft.

11 In seinen Geschichtserzählungen ist von diesem weichen Herz wenig zu merken, denn bezüglich der Menschen beweist er kein Mitleid. Fehlende Moral und Ironie vermischen sich lustvoll. So beschreibt er einen - angeblich in Haiti bekannten, aber fiktiven - 
Piraten Patrice de la Porte als „das schwarze Schaf einer angesehenen Gelehrtenfamilie, gewalttätig, inzestuös und eigenbrötlerisch war dieser Mann, einfach zum Verlieben.“ (M 157) Es wird übertrieben, so sind manche Figuren äußerst bizarr und schillernd gezeichnet. Der genannte Pirat, dem skurrilerweise noch die Erfindung des MolotowCocktails zugesprochen wird, verschont so Leute, die er als LiebhaberInnen und Modelle für seine Bildhauerei verwenden kann oder die ihm ein gutes Rezept verraten. Schwarzer Humor bleibt ein vorherrschendes Stilmittel dieser Geschichtsbetrachtung.

\section{Untrennbare Mischung von Fakten, Mythen, Gerüchten und Legenden}

12 Über historische Figuren erfahren die Lesenden oft mehr Legenden als fundierte Fakten. Erzählt wird beispielsweise von der grausamen „Blutgräfin“ Erzsébet Báthory, die als historische Person ihre Bediensteten sadistisch gequält und getötet hat, dann vermutlich als Opfer einer politischen Intrige von Seiten des Hauses Habsburg dafür gerichtet worden ist. Der Erzähler nennt sie liebevoll Elisa und entschuldigt sie mit ihrer schweren Kindheit und Krankheit. Einerseits übernimmt er dabei die entstandenen Mythen, andererseits bezieht er sich humorvoll auf die Legendenbildungen: „Später entstand die Legende, Elisabeth habe im Blut der ermordeten Mädchen gebadet, es getrunken, was allerdings nicht ganz den Tatsachen entsprach, sie trank am allerliebsten Wein." (M 216)

13 Allwissend ist der Erzähler keineswegs; oft benennt er Mythisierungen und baut sein Wissen explizit auf Gerüchte - so liest man über den Tod des deutschen Afrikaforschers Robert Flegel, der sich angeblich auf einen Skorpion gesetzt hat: „Angeblich war das Tier völlig ungefährlich, aber Flegel starb trotzdem, vielleicht war es einfach nur die Aufregung.“ (M 121)

14 Fakt und Fiktion wollen und sollen im Roman schwer unterscheidbar sein; die Wahrnehmung geschichtlicher Wahrheiten scheint unmöglich: „Ich habe erst unlängst davon erzählt, dass die Dinge manchmal klar scheinen, und später stellt sich heraus, dass es nur Trug war." (M 227) Die Vernetzung von historischen Fakten und imaginierten Ausschilderungen, deren Grenzen fließen und nicht leicht fassbar sind, zeichnen den Roman aus, unter anderem wenn die historisch belegte, erste Heißluftballonfahrt mit Passagieren - und zwar mit Hahn, Hammel und Ente - keine baldigen Nachfolgefahrten hat: „Die Mission galt als voller Erfolg, Ludwig [XVI.] höchstpersönlich verspeiste beim Festbankett die Mannschaft, bekam aber Durchfall, was weitere Versuche erheblich verzögerte.“ (M 26)

Es ist ein Spiel mit der Manipulation der Geschichtsschreibung und dem althergebrachten Glauben an Geschriebenes und Gedrucktes. Welche Aussagen als Fiktion einzuordnen sind und welche als Fakt, kann der Lesende dabei ohne Zuhilfenahme mancher Nachschlagewerke nicht bestimmen: Stavarič gräbt auch historisch belegte, aber erstaunliche Tatsachen aus: so etwa dass die Darstellerin der ersten Nacktszene der Filmgeschichte, Hedy Lamarr, auch ein Patent für eine störungssichere Funkfernsteuerung angemeldet hat, oder dass die Vasa, das erste Schiff mit zwei Kanonendecks und Prestigeobjekt der schwedischen Militärmacht, 1628 während ihrer Jungfernfahrt sofort beim ersten stärkeren Windstoß kenterte. 


\section{Geschichte als Gegenstand einer Konstruktion}

In seiner 14. These formuliert Benjamin: „Die Geschichte ist Gegenstand einer Konstruktion, deren Ort nicht die homogene und leere Zeit sondern die von Jetztzeit erfüllte bildet" ${ }^{\text {"s }}$. Er lenkt damit die Aufmerksamkeit auf die Bedeutung des Moments der Geschichtsschreibung, deren Rezeption und Interpretation.

Inwieweit Geschichte im Nachhinein, aus einer konkreten Zeit und Situation heraus, interpretiert und in ihrer Stimmigkeit und Konsequenz erfunden wird, zeigt sich immer wieder im Roman, so die Auslegung der Ereignisse vor dem Untergang der Titanic: „Eine blonde Frau bekam ihre Tage und klagte über Kopfschmerzen. Später sollten ihre Nachkommen ungeniert behaupten, die Großmutter hätten Vorahnungen geplagt“. (M 42)

Geschichtsmanipulation wird explizit thematisiert, so in Bezug auf Herostratos, der den Tempel der Artemis in Ephesos in Brand setzte, ,um seinen Namen, wie er glaubte, auf alle Ewigkeit unsterblich $\mathrm{zu}$ wissen. Ich meine, der hat wirklich erkannt, was Geschichte bedeutet.“ (M 12) Mit Gewalt hatte er bekanntlich mehr Erfolg als die städtischen Autoritäten, die ein Nennungsverbot seiner Brandstiftung und seines Namens, eine damnatio memoriae, verhängt hatten.

Die von der Kirche gesteuerte Historiographie wird ebenso zum Thema: „Im zwölften Jahrhundert hatte Papst Gregor IX. die glorreiche Idee, jeden Gedanken, der ihm verhasst war, einzudämmen, jede Geschichte, die ihm missfiel, wollte er für alle Zeiten unkenntlich wissen, als hätte sie nie existiert. Er strebte danach, allein über das Maß des Lebens zu richten, das Gute wie das Böse sollte begradigt werden, und viele auf der Welt dachten wie er, dass ein Gott, ihr Gott, die Erde erschaffen habe, er allein, und dass alles ganz einfach sei." (M 15) Begradigung und Vereinfachung werden der Geschichtsschreibung an mehreren Stellen vorgeworfen. Zusammen mit der Mischung aus Fakten und Fiktion ergibt der Roman damit ein Bild der Unsicherheit; die Glaubenswürdigkeit der Historiographie wird relativiert.

Die Rolle der Kirche als geschichtsmanipulierende Kraft, die sich gegen das Wissen und die Kunst stellt, wird öfter angeschnitten, so wenn ein 1726 gefundener fossiler Riesenmolch als Sünder der Sintflut gesehen wird oder wenn Melvilles Moby Dick Kritik erfährt, „angeblich weil er ebenda die traditionelle Religion verspottete, den Götzendienst dem Christentum als ebenbürtig erklärte." Dazu folgt einmal mehr statt Empörung der sarkastische Kommentar des Erzählers: „Ich meine, ob die Menschen nicht ganz andere Sorgen hatten?"“ (M 236)

\section{Große und kleine Geschichten}

21 „Der Chronist, welche die Ereignisse hererzählt, ohne große und kleine zu unterscheiden, trägt damit der Wahrheit Rechnung, daß nichts, was sich jemals ereignet hat, für die Geschichte verloren zu geben ist. ${ }^{\text {" }}$ Diese Feststellung in Benjamins dritter These zielt insbesondere auf die erlöste Menschheit, der ihre Vergangenheit vollauf zufallen werde und die damit jeden ihrer vergangenen Momente zitieren kann.

22 Auf ähnlichen Gedanken basiert die postmoderne Geschichtsauffassung: Nach JeanFrancois Lyotard sind die „großen Erzählungen“, absolute und allgemeingültige Erklärungsprinzipien, zu verwerfen und müssen durch „kleine Erzählungen“, eine 
Vielzahl unterschiedlicher Sprachspiele, ersetzt werden ${ }^{10}$. Stavarič' Roman folgt dieser Forderung. In Magma werden genau diese großen und kleinen Geschichten und Erzählungen vermischt. Nebensächlichkeiten und Skurrilitäten wie Tiere von Prominenten, Mode oder Wilderer werden detailreich besprochen. Die Schwerpunkte und inhaltlichen Wendungen sind ungewohnt und verwundern immer wieder.

Eine lineare Geschichtsauffassung wird unterwandert. Stavarič verweigert sich einem chronologischen, linearen Erzählen, stattdessen werden Erinnerungen, Träume, Gedanken und Erlebnisse der Gegenwart montiert. Die Chronologie geht trotz aller erwähnten Jahreszahlen vollkommen verloren. Stattdessen wird uns ein Netzwerk an geschichtlichen Beziehungen, Assoziationen und Konsequenzen präsentiert. Nicht zufällig wurde dieser Aufbau mit einem Surfen bei Wikipedia und Google verglichen ${ }^{11}$. $\mathrm{Zu}$ dieser Technik bezieht der Autor Stellung, die seine bereits dargestellte Haltung zur Geschichtsschreibung untermauert:

„Das Fragmentarische des Textes spiegelt auch mein Geschichtsverständnis. Ich sehe keinen roten Faden, der sich durch die Zeiten durchexerzieren ließe. Den kann man nur als Schriftsteller konstruieren. Ich arbeite mit Collage und Montage, weil ich die Geschichte nicht als konsequente logische Erzählung sehe. Die Schicksale kommen und gehen. Ich misstraue der Faktizität." ${ }^{12}$

Jahreszahlen werden zwar vielfach verwendet; zu den einzelnen Zahlen - z. B. zu 1906, 1889, 1914 und dann wieder zu 1819 - werden verschiedenste Geschehnisse angeführt und aneinandergereiht, die man in keinen Geschichtsbüchern finden würde. Der Blick der Lesenden wird auf unerwartete Geschehnisse gelenkt, was auf unbekannte Gleichzeitigkeiten, Folgen und Zufälle aufmerksam macht. So erfährt man zum Jahr 1914 nichts von Sarajewo, obwohl des Erzählers Interesse an Attentaten sonst groß ist. Wir lesen nur lapidar, dass sich im Krieg die neu eingesetzten Reißverschlüsse als nützlich erwiesen. Statt mehr vom Weltkrieg erfahren wir von einem Mordfall und dem darauffolgenden Prozess, einen Hochflugweltrekord und von Chaplinfilmen.

Skurrile Sprünge werden aufgrund der Gleichzeitigkeit unternommen, Erzählungen so gebrochen: Ein tödlicher Vulkanausbruch auf Martinique 1902 führt den Erzähler sogleich zur Gründung von Real Madrid im gleichen Jahr.

Zufällige Assoziationen und Zusammenhänge werden erwähnt, so dass der kleine Prinz Saint Exupery vom Asteroiden 612 stammt, der König von Northumbria Oswiu 612 geboren wurde und die vermutliche Serienmörderin Báthory 612 Namen von Ermordeten in ihr Notizbuch notiert haben soll. Damit wird wiederum die Logik der Erwähnung aufgrund der gleichen Jahreszahl relativiert und in Frage gestellt.

\section{Schicksal, Plan und Ziel}

Fragen eines Plans der Geschichte durchziehen implizit und explizit den Roman. Bestimmung und Schicksal werden vom Erzähler immer wieder durchdacht. Ein zielgerichteter, gar absichtsvoller Plan wird dabei durchwegs als Schimäre dargestellt:

„Bestimmung, das ist schon ein starkes Stück, es suggeriert Absicht, wo niemals welche war, kennt keinen Anfang und kein Ende, alles Werden fasst sie in eine Zwischensumme, eine sich ewig fortsetzende Handlung. Der Dämon rechnet und protokolliert, die Geschichten fügen sich, selbst die beiläufigen, die, welche man im Vorbeigehen hört, tragen das Ihre bei. Wenn man nur kurz nachdenkt, wird einem klar, dass nichts zufällig passiert, aber es geht dann doch alles in die Hose." (M 41) 
Zufälle werden negiert, beispielsweise wenn Beethoven an dem Tag sein Gehör verloren haben soll, an dem Offenbach zur Welt kam. Der Fortgang der dargestellten Geschichte scheint einer Maschine ähnlich, deren Bewegungen ineinandergreifen und Konsequenzen haben: Menschen glauben nur, „sie hätten sich aus freien Stücken entschieden, die Kolben, die Rädchen, die Zähnchen der Welt.“ (M 230) Die Rädchen der Maschine der Geschichte erlauben weder Zufälle noch Selbstbestimmung. Die Rolle der Menschen liegt dabei eben nicht in der erfolgreichen Umsetzung ihres freien Willens, sondern in ihrer Mitschuld: „Dabei ist niemand frei von Schuld, es gibt kein ungerechtes Schicksal, es gibt die Bestimmung und mich, jemanden wie mich sollte man nicht unterschätzen.“ (M 44)

Dass das erstarkte Selbstbewusstsein der Moderne mit ihren Ideen des geschichtslenkenden Subjekts grundlos war, ist dem Erzähler klar: „Die Moderne erfand sich irgendwann den Zufall als Bestandteil der Realität, alles war plötzlich ein Akt der Selbstbestimmung. Ich habe mich bestimmt, aber was genau passiert morgen?" (M 40) Niemand kann den Lauf der Welt im Roman lenken, selbst der Teufel nicht: „ich schreibe Geschichte, nur das eigene Ende vermag ich nicht abzusehen.“ (M 173)

Das Individuum kann im Roman also nur auf die Einflüsse und Eindrücke der Welt reagieren, seine eigene Zukunft keineswegs bestimmen. Nicht einmal der teuflische Erzähler hat das Schicksal in der Hand; er scheint Benjamins Engel der Geschichte nicht nur in den Formulierungen - sehr ähnlich zu sein. Er will wohl mitbestimmen, bleibt aber machtlos. „Ich möchte mich an alles erinnern, was war und was hätte sein können, das Schicksal spielen, das Schicksal herausfordern, vielleicht einmal noch die Welt antreiben mit eiserner Faust, wie in den guten, blutigen Tagen. Ich will Geschichte schreiben, selbst jetzt noch, da ich längst müde bin.“ (M 242)

Die Maschine Geschichte, welche keine Zufälle zulässt, scheint keineswegs einem Plan oder Ziel zu entsprechen, sondern vielmehr der Chaostheorie, in dessen vollkommen dynamischem System sich die Elemente zwar beeinflussen, aber die Konsequenzen nicht vorhersagbar sind. Eine Lösung wird nur in der Akzeptanz dieses Chaos angedacht: „Es heißt, dass man sich von der Verdammnis befreien kann, wenn man sein eigenes Schicksal annimmt.“ (M 130)

Wie schwer es ist, sich eine Vorstellung von Geschichte ohne Plan und Ziel zu machen, zeigt der Erzähler, der dies selbst nur langsam begreifen kann: „Mir kamen erste Zweifel, nach so vielen Jahren gelangte ich an den Punkt, wo ich mich fragen musste, was, wenn es gar keinen Plan gibt, keinen Zusammenhalt in diesen ganzen Geschichten.“ (M 116)

\section{Kritik an der Vorstellung des Fortschritts überhaupt}

„Die Kritik an der Vorstellung dieses Fortgangs [,der eine homogene und leere Zeit durchläuft,] muß die Grundlage der Kritik an der Vorstellung des Fortschritts überhaupt bilden, ${ }^{~}{ }_{13}^{13}$ so Benjamin. In der 13. These entwirft er die notwendige Fundierung einer theoretischen Kritik am Fortschrittsbegriff, dessen Überhöhung als unaufhaltsamer und unabschließbarer Fortschritt der Menschheit Benjamin fatal erscheint.

Illusion, wobei dies erneut mit postmodernen Erkenntnissen übereinstimmt. Dass diese 
Überzeugung fälschlicherweise von der bewiesenen Evolution der Pflanzen- und Tierwelt stammt, macht der Erzähler nachvollziehbar, wenn am Beginn eine Schöpfungsgeschichte erzählt wird - von einer Zeit, „in der ich eins war mit dem Universum, ich wollte jeden und alles im Fortschritt wissen, mich selbst an allem messen, ich meine, wer kann das schon von sich behaupten?" (M 8) Die ironische Brechung am Absatzende fehlt auch hier nicht.

Der Erzähler ähnelt somit Benjamins Engel der Geschichte, der statt einer Kette von Begebenheiten „eine einzige Katastrophe, die unablässig Trümmer und Trümmer häuft", sieht. Selbst die titelgebende Gesteinsschmelze bei vulkanischen Tätigkeiten kann als Sinnbild für Vernichtung gelesen werden. Zerstörende Kräfte der Geschichte werden beleuchtet: Schiffskatastrophen, Kriege, Umstürze und Attentate - auch innerhalb der Familie - bestimmen das Bild. Es ist kein Geschichtsbild des Optimismus, kein Geschichtsbild der Lehrbücher, in denen sich die Menschheit in Richtung größere Freiheit, Wohlstand und Mitbestimmung bewegt: „Nach all den Jahren bleibt die Frage, ob sich der Lauf der Welt irgendwann bessert." (M 52) Die erwähnte Nichtzufälligkeit der Geschichte ist also nicht teleologisch gelesen, sondern diabolisch: als Katastrophengeschichte. Die - in der bereits erwähnten Maschine Geschichte ablaufenden Begebenheiten verursachen Konsequenzen, meist desaströse. Der Roman illustriert diese Idee fortwährend, zum Beispiel wenn für den Nil einsame Mädchen geopfert wurden und das Leid im Wasser gespeichert blieb: „Die Geschichte hinterlässt ihre Spuren.“ (M 9) Diese Spuren sind schrecklich, Fehler, Irrtümer und Brutalitäten treiben die Geschichte voran: „die Geschichte ist ein komplexes Gebilde, ein falscher Schritt führt zum nächsten“. (M 218)

Eine kontinuierliche Aufwärtsentwicklung der Menschheit kann demnach nicht stattfinden. Der falsche Glauben an den und die Kritik am Fortschritt beweist sich subtil als roter Faden des Romans. „Ich meine, der Fortschritt wurde irgendwie allen zum Verhängnis.“ (M 198) So sind es auch nicht die uns gewohnten Erfolgsgeschichten der Modernen, die Fortschritt vermittelnden Erfinder und Entdecker, auf die der Erzähler unseren Blick lenkt, sondern deren Rückschläge und Katastrophen. Nicht einmal erfreuliche Erfindungen finden Lob, denn „,niemand konnte damals ahnen, wohin der führt, wozu er eigentlich gut ist, der Fortschritt.“ (M 73) Aus der Geschichte der Elektrizität, ein Sinnbild des Fortschritts schlechthin, lesen wir vor allem von historischen Stromausfällen; als signifikante Erfindungen finden der elektrische Stuhl und das Schwarzpulver Erwähnung; bei pharmazeutischen Mitteln erfahren wir die vernichtenden Nebenwirkungen, so wie beim Schlafmittel Contergan, das Missbildungen bei Neugeborenen hervorrief, und beim Stärkungsmittel Frauengold, das wegen krebserregender Wirkstoffe verboten wurde; Dosen wurden angeblich vor brauchbaren Dosenöffnern erfunden. Symbolisch wird auch der historisch reale Tod des erfolgreichen Erfinders Thomas Midgley, Jr. beschrieben ${ }^{14}$. Er verhedderte und strangulierte sich nämlich in seinem System von Schnüren und Umlenkrollen, das ihn als Behinderten aus dem Bett heben sollte.

Der Fortschritt scheint nur im Interesse weniger zu liegen; er erscheint als leere Floskel zur Mobilmachung im Krieg und zur Disziplinierung: Die Kabellegung über den Atlantik erscheint nicht im Licht schnellerer Kommunikationsmöglichkeiten, sondern: „es galt im Namen des Fortschritts, ganze Kontinente an die Leine zu legen.“ (M 124)

Die Fortschrittsgläubigkeit zeigt sich sogar als das eigentliche Böse, wobei an das unheilvolle Faustische Streben erinnert wird: 
„Wenn mich einer fragt, der wahre Dämon bleibt die Neugier, der unersättliche Hunger nach den zahllosen flüchtigen Erscheinungen des Universums. Man lebt fortan mit der Lust, sie zu katalogisieren, ihnen die Flüchtigkeit zu nehmen, ihnen ihr Geheimnis zu rauben, alles in ein absonderliches, systematisches Museum zu verwandeln. Ich glaube, das nennt man Fortschritt.“ (M 99)

Der Roman liefert auch den Grund, warum der menschliche Fortschritt scheitert, sich von der aufwärtsstrebenden Evolution der Pflanzen und Tierwelt abkoppelt. Der Mensch scheint des Menschen Hölle, und das verunmöglicht eine Verbesserung. Sei es beim Stromausfall 1977 in New York, der (auch historisch) zu Plünderungen und Bränden führte, oder sei es beim immer wieder beschriebenen Unglück der Titanic, wo sich die Sinnlosigkeit menschlichen Tuns und Denkens kurz vor dem Untergang im Streit der Kinder, wer im Etagenbett oben und unten liegen dürfe, zeigt. Die Menschen tragen hier die Schuld an dem apokalyptischen Ereignis: Warnungen vor den Eisbergen wurden vom Funker ignoriert, damit er hunderte wichtigtuerische Telegramme der Passagiere zügig senden konnte. Um die Rettungsboote kämpften später Engländer und Italiener. Der Erzähler weiß von 497 freien Plätzen in Rettungsbooten, in denen Seemannslieder gesungen wurden, um die Hilferufe der Ertrinkenden zu übertönen. Robbenfänger retteten die Schiffsbrüchigen nicht, um nicht nicht aufgrund ihrer illegalen Tätigkeit in Schwierigkeiten zu kommen.

Andere Schiffsbrüche und deren schreckliche Konsequenzen können im Roman genauso auf den menschlichen Egoismus zurückgeführt werden - so etwa, wenn beim Skandal um das französische Schiff Méduse Offiziere in Beibooten die Stricke zu ihrer Mannschaft auf Flößen durchschneiden, um schneller voranzukommen oder wenn Kannibalismus nur die Stärkeren überleben lässt.

Der Mensch wird so keineswegs als Höhepunkt einer Evolution dargestellt, sondern vielmehr als ein „falscher Schritt“, der ständig weitere falsche Schritte provoziert.

\section{Fazit}

Es konnte gezeigt werden, dass der Roman Magna sich nicht nur vordergründig mit der Erzählung von Geschichte beschäftigt, sondern auch metahistoriographische und geschichtsphilosophische Positionen einnimmt. Eine Übersicht dieser Stellungnahmen zeigt eine starke Inspiration durch Walter Benjamins Thesen über den Begriff der Geschichte, aus welchen auch ein - zwar gebrochenes - Zitat dem Roman vorangestellt ist, wobei der Roman gerade auf die marxistische Ideologie und den messianischen Glauben Benjamins verzichtet, was vor allem mit der zeitlichen Distanz zu begründen ist. Doch auch Stavarič' Biographie mag dabei eine Rolle spielen, denn seine Familie entfloh der kommunistischen Tschechoslowakei.

Die Geschichtsschreibung wird im Roman - vor allem mit dem Stilmittel des Humors hinsichtlich ihrer Moral, ihrer Faktizität und ihres konstruierten Charakters diskutiert. Ausgehend von Benjamins Haltung nähert sich der Roman postmodernen Positionen an. Die Reihungen von Geschichten und Erinnerungen lassen keine große Erzählung zu und verweigern es, einen Plan, einen Fortschritt oder ein Ziel in der Geschichte wahrzunehmen. Die menschliche Historie wird als „einzige Katastrophe“ gezeigt.

Das 23. und letzte Kapitel, der Epilog für die Verdammten, verkündet drei Anklagen. Verurteilt wird dabei der Glaube an „große Erzählungen“ - an Selbstbestimmung und Eigenmächtigkeit, an Fortschritt und Verbesserung, an Vernunft und Wahrheit: „Einer 
hat an die eigene Macht geglaubt / er muss es bezahlen mit seinem Haupt. // Ein anderer hoffte auf ein besseres Morgen / und erwachte im Heute mit steten Sorgen. // Einer wollte mit der Wahrheit ein Kind, / dafür sollte er hängen im Wind.“ (M 243) (Danach werden übrigens - typisch für Stavarič' ironische und Kapriolen schlagende Erzählhaltung - die Richter aufgehängt.)

Nicht nur auf diese Weise schlägt Magma eine alternative, pessimistische Geschichtssicht voller kleiner fragmentarischer Ereignisse und Unglücke vor, die einer optimistischen Idee von Fortschritt und Aufwärtsbewegung der menschlichen Geschichte diametral entgegensteht.

\section{NOTES}

1. Z. B. Krimbacher, Elisabeth, „Michael Stavarič: Normal sind eh die Anderen“ in: PresseSchaufenster, 22.06.2007. (http://diepresse.com/home/kultur/literatur/312090/index.do-Zugriff 30.06.2012).

2. Diese Einführung findet sich ähnlich in meinem Beitrag, der die drei ersten Romane Stavarič' stillborn, Terminifera und Magma - stilistisch in die Epoche der Postmodernen einordnet. Vgl. Lughofer, Johann Georg, „Postmodernität in der zeitgenössischen österreichischen Literatur. Eine Analyse anhand der frühen Romane von Michael Stavarič“ in: Germanistische Beiträge 2010, 27, S. 78-110.

3. Stavarič, Michael, Magma, Salzburg, Residenz 2008, S. 5 - in weiterer Folge mit der Sigle M und Seitenzahl in Klammer im Text genannt.

4. Benjamin, Walter, Erzählen. Schriften zur Theorie der Narration und zur literarischen Prosa, Frankfurt am Main, Suhrkamp, 2007, S. 133.

5. Vgl. Bulthaup, Peter (Hrsg.), Materialien zu Benjamins Thesen „Über den Begriff der Geschichte“, Beiträge und Interpretationen, Frankfurt am Main, Suhrkamp, 1975 und Schweppenhäuser, Hermann, Zum Geschichtsbegriff Walter Benjamins in: Karl Rahner Akademie (Hrsg.), Geschichte denken, Münster, LIT, 1999, S. 95-108.

6. Benjamin, Erzählen, S. 132.

7. Die Hervorhebungen durch Kursivierungen in diesem wie in den folgenden Zitaten sind im Original.

8. Benjamin, Erzählen, S. 137.

9. Ebd., S. 130.

10. Vgl. Lyotard, Jean-Francois, Das postmoderne Wissen. Ein Bericht. Wien, Passagen Verlag, 2009.

11. Z. B. Weiss, Christian, „Der Wikipedia-Mephisto“ in Zeit Online, 29.12.2008 - (http:// www.zeit.de/online/2008/52/michael-stavaric-magma - Zugriff am 30.06.2012) oder Renöckl, Georg, „Michael Stavarič: Magma“ in: http://www.literaturhaus.at/index.php? $\mathrm{id}=3411 \& \mathrm{~L}=0 \% 252 \mathrm{~F} / \mathrm{-}$ Zugriff am 30.06.2012.

12. Mayer, Norbert, „Michael Stavarič: Ohne Katastrophe kein Fortschritt“ in: Die Presse, 7.10.2008 - (http://diepresse.com/home/kultur/literatur/420725/index.do - Zugriff am 30.06.2012).

13. Benjamin, Erzählen, S. 136.

14. Midgley wurde übrigens außerhalb des Romans anklagenderweise mehr Auswirkung auf die Atmosphäre zugeschrieben als jedem anderen Organismus in der Erdgeschichte. Vgl. McNeil, J.R., 
Something New Under the Sun: An Environmental History of the Twentieth-Century World, New York, W. W. Norton \& Company, 2001.

\section{RÉSUMÉS}

Der Roman Magma wird hinsichtlich seiner Geschichtsbetrachtung untersucht. Dabei werden die - bereits im Motto des Romans erwähnten - Thesen Über den Begriff der Geschichte Walter Benjamins als Folie verwendet, wobei gezeigt werden kann, dass und wie deren Hauptaussagen in Magma eine literarische Umsetzung finden. Der Roman geht aber über diese Konzepte hinaus und nähert sich einer postmodernen Haltung an, indem er sich explizit „großen Erzählungen“ verweigert. Stattdessen wird eine alternative, pessimistische Geschichtssicht voller kleiner fragmentarischer Ereignisse präsentiert, die einer optimistischen Idee von Fortschritt und Aufwärtsbewegung der Menschheit diametral entgegensteht.

Cet article s'intéressera au roman Magma d'un point de vue historique. Les concepts de Walter Benjamin présentées dans ses Thèses sur le concept d'histoire, qui servent d'épigraphe au roman, forment le cadre conceptuel illustrant l'interprétation littéraire de ces thèses et la manière dont elles sont mises en pratique. Mais le roman va plus loin et adopte une position postmoderne en refusant explicitement les "grandes histoires». Elles sont remplacées par un regard autre, pessimiste sur l'histoire caractérisé par des événements divers et dispersés, s'opposant de la sorte à une vision optimiste du développement et du progrès de l'humanité.

This paper examines the novel Magma from a historical point of view. The basic concepts of Walter Benjamin's Theses on the Philosophy of History, which are already mentioned in the themes of the novel, provide a conceptual framework illustrating both the literary interpretation of his theses in Magma and the way they are implemented. However, the novel goes beyond Benjamins's theses and comes near a post-modern position by explicitly denying the value of "grand narratives". Instead, it presents an alternative, pessimistic outlook on history characterized by various scattered events, thereby diametrically opposing an optimistic idea of human progress and development.

\section{AUTEURS}

\section{JOHANN GEORG LUGHOFER}

Universität Ljubljana (Slowenien) 